\title{
Validation of the Publication of New Names and New Combinations Previously Effectively Published Outside the IJSB
}

\author{
List No. $19 \dagger$
}

The purpose of this announcement is to effect the valid publication of the following new names and new combinations under the procedure described previously (Int. J. Syst. Bacteriol. 27(3):iv, 1977). Authors and other individuals wishing to have new names and/or combinations included in future lists should send the pertinent reprint or a photocopy thereof to the IJSB for confirmation that all of the other requirements for valid publication have been met. It should be noted that the date of valid publication of these new names and combinations is the date of publication of this list, not the date of the original publication of the names and combinations. The authors of the new names and combinations are as given below, and these authors' names will be included in the author index of the present issue and in the volume author index in this issue of the IJSB.

Inclusion of a name on these lists validates the name and thereby makes it available in bacteriological nomenclature. The inclusion of a name on this list is not to be construed as taxonomic acceptance of the taxon to which the name is applied. Indeed, some of these names may, in time, be shown to be synonyms, or the organism may be transferred to another genus, thus necessitating the creation of a new combination.

\begin{tabular}{|c|c|c|c|}
\hline Name & Proposed as: & Author(s) (reference) & Nomenclatural type ${ }^{a}$ \\
\hline Campylobacter hyointestinalis & New species & Gebhart et al. (2) & Strain 80-4577-4 $(=\text { ATCC } 35217)^{b}$ \\
\hline Eubacterium angustum & New species & Beuscher and Andreesen (1) & Strain MK-1 (= DSM 1989) \\
\hline Fervidobacterium & New genus & Patel et al. (6) & F. nodosum ${ }^{c}$ \\
\hline Fervidobacterium nodosum & New species & Patel et al. (6) & Strain Rt17-B (= ATCC 35602) ${ }^{b}$ \\
\hline Kitasatosporia griseola & New species & Takahashi et al. (7) & Strain AM-9660 $(=\text { IFO } 14371=\text { JCM 3339 })^{b}$ \\
\hline Kitasatosporia phosalacinea & New species & Takahashi et al. (7) & Strain KA-338 $(=\text { IFO } 14372=\text { JCM } 3340)^{b}$ \\
\hline Marinococcus & New genus & Hao et al. (3) & M. halophilus ${ }^{b}$ \\
\hline Marinococcus albus & New species & Hao et al. (3) & 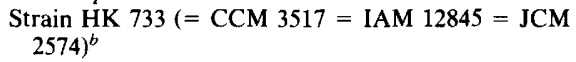 \\
\hline $\begin{array}{l}\text { Marinococcus halophilus } \\
\text { (basonym: Planococcus } \\
\text { halophilus) }\end{array}$ & New combination & $\begin{array}{l}\text { (Novitsky and Kushner) Hao et } \\
\text { al. (3) }\end{array}$ & $\begin{array}{l}\text { ATCC } 27964(=\text { Strain HK } 718=\text { CCM } 2706= \\
\text { IAM } 12844=\text { JCM 2479) }\end{array}$ \\
\hline Methanosphaera & New genus & Miller and Wolin (5) & M. stadtmanaeb \\
\hline $\begin{array}{l}\text { Methanosphaera stadtmanae } \\
\text { (M. stadtmaniae }[\mathrm{sic}])\end{array}$ & New species & Miller and Wolin (5) & Strain MCB-3 (= DSM 3091) \\
\hline Microbacterium arborescens & $\begin{array}{l}\text { Revived name as new } \\
\text { combination }\end{array}$ & Imai et al. (4) & ATCC $4358(=\text { IFO 3750 })^{b}$ \\
\hline
\end{tabular}

$\dagger$ Lists 1 through 18 were published in the Int. J. Syst. Bacteriol. 27:306, 1977; 29:79, 1979; 29:436, 1979; 30:601, 1980; 30:676, 1980; 31:215, 1981; 31:382, 1981; 32:266, 1982; 32:384, 1982; 33:438, 1983; 33:672, 1983; 33:896, 1983; 34:91, 1984; 34:270, 1984; 34:355, 1984; 34:503, 1984; 35:223, 1985; and 35:375, 1985.

${ }^{a}$ Abbreviations: ATCC, American Type Culture Collection, Rockville, Md.; CCM, Czechoslovak Collection of Microorganisms, Brno, Czechoslovakia; DSM, Deutsche Sammlung von Mikroorganismen, Göttingen, Federal Republic of Germany; IAM, Institute of Applied Microbiology, University of Tokyo, Tokyo, Japan; IFO, Institute for Fermentation-Osaka, Osaka, Japan; JCM, Japan Collection of Microorganisms, Wako, Japan.

${ }_{b}^{b}$ Type designated by the authors.

c Type by monotypy.

${ }^{d}$ Type previously established (Approved Lists of Bacterial Names, Int. J. Syst. Bacteriol. 30:225-420, 1980).

\section{LITERATURE CITED}

1. Beuscher, H. U., and J. R. Andreesen. 1984. Eubacterium angustum sp. nov., a gram-positive anaerobic, non-sporeforming, obligate purine fermenting organism. Arch. Microbiol. 140:2-8.

2. Gebhart, C. J., P. Edmonds, G. E. Ward, H. J. Kurtz, and D. J. Brenner. 1985. "Campylobacter hyointestinalis" sp. nov.: a new species of Campylobacter found in the intestines of pigs and other animals. J. Clin. Microbiol. 21:715-720.

3. Hao, M. V., M. Kocur, and K. Komagata. 1984. Marinococcus gen. nov., a new genus for motile cocci with meso-diaminopimelic acid in the cell wall; and Marinococcus albus sp. nov. and Marinococcus halophilus (Novitsky and Kushner) comb. nov. J. Gen. Appl. Microbiol. 30:449-459.

4. Imai, K., M. Takeuchi, and I. Banno. 1984. Reclassification of
"Flavobacterium arborescens" (Frankland and Frankland) Bergey et al. in the genus Microbacterium (Orla-Jensen) Collins et al., as Microbacterium arborescens comb. nov., nom. rev. Curr. Microbiol. 11:281-284.

5. Miller, T. L., and M. J. Wolin. 1985. Methanosphaera stadtmaniae [sic] gen. nov., sp. nov.: a species that forms methane by reducing methanol with hydrogen. Arch. Microbiol. 141:116-122.

6. Patel, B. K. C., H. W. Morgan, and R. M. Daniel. 1985. Fervidobacterium nodosum gen. nov. and spec. nov., a new chemoorganotrophic, caldoactive, anaerobic bacterium. Arch. Microbiol. 141:63-69.

7. Takahashi, Y., Y. Iwai, and S. Ōmura. 1984. Two new species of the genus Kitasatosporia, Kitasatosporia phosalacinea sp. nov. and Kitasatosporia griseola sp. nov. J. Gen. Appl. Microbiol. 30:377-387. 